\title{
Dynamic lot sizing and tool management in automated manufacturing systems
}

\author{
M. Selim Akturk*, Siraceddin Onen \\ Department of Industrial Engineering, Bilkent University, 06533 Bilkent, Ankara, Turkey
}

Received 1 September 1999; received in revised form 1 June 2000; accepted 1 November 2000

\begin{abstract}
The overall aim of this study is to show that there is a critical interface between the lot sizing and tool management decisions, and these two problems cannot be viewed in isolation. We propose five alternative algorithms to solve lot sizing, tool allocation and machining conditions optimization problems simultaneously. The first algorithm is an exact algorithm which finds the global optimum solution, and the others are heuristics equipped with a look-ahead mechanism to guarantee at least local optimality. The computational results indicate that the amount of improvement is statistically significant for a set of randomly generated problems. The magnitude of cost savings is dependent on the system parameters.
\end{abstract}

\section{Scope and purpose}

In most of the studies on tool management, lot sizes are taken as a predetermined input while deciding on tool allocations and machining parameters. This might create empty feasible solution spaces and otherwise unnecessarily limit the number of alternatives possible for the tool management problem. In this study, we consider the integration of lot sizing and tool management problems to minimize total production cost for multiple periods under dynamic demand. By integrating these decisions we not only improve the overall solution, but also prevent any infeasibility that might occur for the tool management problem due to decisions made at the lot sizing level. (C) 2002 Elsevier Science Ltd. All rights reserved.

Keywords: Flexible manufacturing systems; Lot sizing; Tool management

* Corresponding author. Tel.: + 90-312-266-4477; fax: + 90-312-266-4054.

E-mail address: akturk@bilkent.edu.tr (M.S. Akturk). 


\section{Nomenclature}

\section{Parameters}

$\alpha_{j}, \beta_{j}, \gamma_{j} \quad$ speed, feed, depth of cut exponents for tool $j$

$C_{\mathrm{m}}, b, c, e$ specific coefficient and exponents of the machine power constraint

$C_{0} \quad$ operating cost of the CNC machine $(\$ / \mathrm{min})$

$C_{\mathrm{s}}, g, h, l \quad$ specific coefficient and exponents of the surface roughness constraint

$C_{\mathrm{t}_{j}} \quad$ cost of tool $j(\$ /$ tool $)$

$d_{i p} \quad$ depth of cut for operation $i$ of part $p$ (in)

$D_{p t} \quad$ demand for part $p$ in period $t$ (parts)

$G_{i p}$ diameter of the generated surface for operation $i$ of part $p$ (in)

$h_{p t}$

$H P$

$I_{p}$

$J$ inventory holding cost of part $p$ in period $t(\$ /$ part $/$ period $)$ maximum available machine power (hp)

set of the available tool types

$\sigma_{i} \quad$ set of alternative tool types that can be used for operation $i$

$L_{i p} \quad$ length of the generated surface for operation $i$ of part $p$ (in)

$M \quad$ a very large positive number

$\mathrm{MH}_{t} \quad$ maximum available machine hour in period $t(\mathrm{~min})$

$N_{j t} \quad$ number of available tools of type $j$ in period $t$

$P \quad$ set of all parts

$r_{j} \quad$ tool switching time for tool $j$ (min)

$S F_{i p} \quad$ maximum allowable surface roughness for operation $i$ of part $p(\mu$ in)

$S_{p t} \quad$ setup cost for production of part $p$ in period $t(\$)$

$T \quad$ set of all periods

$T C_{j} \quad$ Taylor's tool life constant for tool $j$

$t s_{p t} \quad$ setup time for production of part $p$ in period $t(\mathrm{~min})$

\section{Decision variables}

$f_{i j p t}$

$I N_{p t}$

$n_{i j p t}$

$q_{i j p t}$

$Q_{p t}$

$t_{\mathrm{m}_{i j p t}}$

$T_{i j p t}$

$U_{i j p t}$

$v_{i j p t}$

$x_{i j p t}$

$Y_{p t}$ feed rate for operation $i$ of part $p$ using tool $j$ in period $t$ (ipr)

inventory level of part $p$ at the end of period $t$ (parts)

number of tool type $j$ required for completion of operation $i$ of part $p$ in period $t$ number of times that an operation $i$ of part $p$ can be performed by a tool type $j$ in period $t$

lot size for part $p$ in period $t$

machining time of operation $i$ of part $p$ using tool $j$ in period $t(\min )$

tool life of tool $j$ in operation $i$ of part $p$ in period $t$ (min)

usage rate of tool $j$ in operation $i$ of part $p$ in period $t$

cutting speed for operation $i$ of part $p$ using tool $j$ in period $t$ (fpm)

$0-1$ binary decision variable which is equal to 1 , if tool $j$ is assigned to operation $i$ of part $p$ in period $t$

$0-1$ binary decision variable which is equal to 1 if $Q_{p t}>0$ 


\section{Introduction}

Material requirements planning (MRP) and manufacturing resource planning (MRP II) have been the most widely implemented large-scale production management systems since the early 1970s. Several thousand systems of this style are in use in industry around the world. MRP-based systems provide an effective framework for managing production. Nevertheless, there are several key weaknesses related to the determination of lot sizes and capacity requirements in these systems. Lot sizing is the only procedure in the MRP system that involves decision making rather than information gathering. As a result, there are alternatives to consider, and different approaches to lot sizing can lead to different plans and performance. Therefore, it would be desirable to optimize the choice of lot sizes. In a standard MRP II system, rough cut capacity planning and capacity requirements planning modules are simply information gathering procedures, and they do not allocate scarce resources when the material plan violates capacity constraints. Instead they just signal the need to revise the master schedule or to increase capacity as stated by Baker [1]. Moreover, the planning decisions are made sequentially such that material plans are made first; then capacity is considered, and an iterative search for a viable schedule follows. Kimms [2] illustrates some of the MRP II shortcomings and proposes a genetic algorithm to solve a joint lot sizing and scheduling algorithm.

MRP II packages are extensively used in manufacturing industry. Approximately $50 \%$ of US annual expenditures on manufacturing is in the metal-working industry, and two-thirds of metal-working is metal cutting. In addition, approximately $75 \%$ of the dollar volume of metalworked products is manufactured in batches of less than 50 parts as reported in Stecke [3]. The growth of the metal-working industry spawned technological improvements over time to improve the productivity of these mid-volume systems, such as the use of computer numerically controlled (CNC) machines and flexible manufacturing systems (FMSs). In view of the high investment and operating costs of CNCs and hence FMSs attention should be paid to their effective utilization. Gray et al. [4] give an extensive survey on the tool management issues of automated manufacturing systems, and emphasize that the lack of tooling considerations has resulted in the poor performance of these systems. In Kouvelis [5], the cost of tooling has been reported to be $25-30 \%$ of the fixed and variable cost of production. Gray et al. also present an integrated conceptual framework for resource planning to examine how tool management issues can be classified into tool, machine and system levels. In this study authors pointed out that efforts in tool management focus on single-level decisions and ignore the impact across the levels which may lead to suboptimal results by stating the integration of decisions at different levels in tool-related research areas. Most of the existing studies in tool management ignore the lot sizing decision at the system level and take it as a predetermined input while deciding on tool allocation and machining parameters. In an automated manufacturing environment, operational problems, such as machining conditions, tool availability, tool life and machine capacity limitations, should be taken into account for the reliable modeling of $\mathrm{CNCs}$, or the absence of such crucial constraints may lead to infeasible results. We can easily claim that significant cost savings can be realized by controlling production rates. Consequently, total production cost can be decreased and any infeasibility due to machine capacity limitations can be avoided.

Lot sizing problems play an important role in production planning systems. Therefore, in a variety of forms it has received much attention in the literature. Wagner and Whitin [6] 
developed a well known $O\left(T^{2}\right)$ dynamic programming algorithm for an uncapacitated model, whereas there are several heuristic procedures such as the least period cost (Silver-Meal), least unit cost, part period balancing and marginal cost difference for the same problem as summarized in Baker [1]. For capacitated problems the situation is quite different. Bitran and Yanasse [7] have shown that the single item capacitated lot sizing problem (CLSP) is NP-hard. Multi item CLSP is also NP-hard except for a few special cases, e.g. when all setup costs are zero. Therefore, most of the literature on this problem focuses on heuristic solution procedures. The heuristic approaches reported in the literature are classified by Kirca and Kokten [8] into two groups as mathematical programming and common sense approaches. The heuristics suggested by Thizy and Van Wassenhove [9] and Trigeiro [10] are Lagrangian relaxation based procedures and belong to the first class. Most common sense heuristics solve the CLSP on a period by period basis. In each period, lot sizes for all items are determined on the basis of a cost savings criterion. In a given period, future demand of items are scheduled to be produced in that period until no further cost savings are possible or until all of the capacity at that period is exhausted, e.g. Dixon and Silver [11]. Unfortunately, in these heuristic procedures, unit production costs and resource consumption rates are assumed to be fixed and a priori known, whereas these are significant decision variables in a machining environment.

For solving the tool allocation problem at the system level, most of the published studies use 0-1 binary variables, i.e. a particular tool $j$ is assigned to operation $i$, to represent tool requirements such as Stecke [3] and Sarin and Chen [12]. At the machine level, most of the studies emphasize the minimization of tool switches due to a change in a part mix $[5,13]$. Unfortunately, all of these studies assume constant lot sizes, production rates as well as processing times. Furthermore, these studies determine the tool requirements for each operation independently, and fail to relate the contention among the operations for a limited number of tools. Also, in the multiple operation case, non-machining time components, such as the tool replacements due to tool wear, can have a significant impact on the total cost of production and the throughput of parts as shown by Tetzlaff [14]. Gray et al. [4] reported that tools are changed 10 times more often due to tool wear than to part mix because of the relatively short tool lives of many turning tools. One exception in the tool management area, where tool wear and tool change issues receive active consideration is perhaps the work of Avci and Akturk [15]; they, however, do not address the lot sizing dimension.

In the literature there exist few studies on the integration of lot sizing and tool management problems. Wysk et al. [16] introduce lot size considerations in determining the optimal cutting speed in a single item, single machine, single-period problem. Koulamas [17] presents a queueing model for determining analytically the optimal lot size in a machining economics problem under stochastic tool life considerations. Koulamas [18] proposes an iterative procedure for the simultaneous determination of the cutting speed and lot size values in a machining system for single- and multiple-part cases using the Lagrangian technique, while the feed rate is taken as a constant. In this study, parts are assumed to be composed of single operation. Consequently, parts are machined by a single cutting tool and tool allocation decisions are not considered. The author also has not considered machine horsepower, surface finish and tool availability constraints, although in many real-life problems the machining parameters are constrained by these limitations. Furthermore, in all of these studies only the single-period case is considered and consequently the demand is assumed to be fixed. 
The overall aim of this study is to show that there is a critical interface between the lot sizing and tool management decisions, and these two problems cannot be viewed in isolation. We propose five alternative solution procedures to find lot sizes, tool allocations and machining parameters simultaneously by integrating system, machine and tool level decisions for a multiple part, multiple operation, multiple-period problem over a single CNC machine to satisfy a dynamic demand. The remainder of this paper is organized into six sections as follows. In the next section, we discuss the underlying assumptions and introduce a mathematical model of the problem. The proposed algorithms are described in Section 3. A numerical example and the computational results are presented in Section 4 and Section 5, respectively. Finally, some concluding remarks are provided in Section 6.

\section{Problem definition}

Consider an automated machining environment consisting of a single CNC turning machine. We are required to solve lot sizing and tool management problems simultaneously to determine the following decision variables: (i) lot sizing decisions, i.e. in what quantities each part will be produced, (ii) tool allocation, i.e. how tools will be allocated to parts in terms of quantities and allocation scheme, and (iii) machining conditions selection, i.e. what the cutting speed and feed rate will be for each operation of each part. The following assumptions are made to define the scope of the study. There are multiple periods and in each period multiple parts are produced. There is a deterministic, but time-varying, demand for every part in every period. Each part is composed of multiple operations and each operation can be performed by a set of alternative tool types from a variety of available tool types with limited quantities on hand. Backlogging is not allowed, and initial and final inventory levels are assumed to be zero. For the machining operations, the cutting speed and the feed rate will be taken as the decision variables, and the depth of cut is assumed to be given as an input. Finally, the $\mathrm{CNC}$ machine can work for a limited number of hours.

Let us demonstrate these interrelated decision making problems on a single part. For each operation of this part, we should decide on which cutting tool will be assigned to perform this operation. Based on CNC machine, part and tool characteristics as well as other manufacturing cost-related data, we will calculate the machining conditions for each operation-tool pair. Machining conditions selection decisions will directly specify the required machining times, tool usage rates, number of cutting tools required to produce all the parts in a given lot size, number of tool changes, and eventually production rates. Since there are limited number of cutting tools on hand for each cutting tool type, different operations will compete for them, which is known as tool allocation problem. Furthermore, the available time of the $\mathrm{CNC}$ machine is also limited. We will formulate a capacitated lot sizing problem to solve these interrelated problems simultaneously, and to show that significant cost savings can be realized by controlling production rates as described below. The notation used in the proposed mathematical model is given under Nomenclature.

The total production cost should include machining time, non-machining time and tooling cost in addition to the setup and inventory holding costs. Machining time, $t_{\mathrm{m}_{i j}}$, is the time that the tool actually engaged in machining to complete a turning operation. Tool life, $T_{i j p}$, is generally defined as the machining time in minutes taken to produce a given wear land for a set of machining conditions. The relationship between the tool life and the machining conditions can be expressed 
as a function of the machining conditions by using an extended form of the Taylor's tool life equation. For the turning operation, a new expression is defined for the machining time to tool life ratio, which is called as the usage rate of tool $j$ in operation $i$ of part $p$ in period $t$, and is denoted by $U_{i j p t}$.

$$
U_{i j p t}=\frac{t_{\mathrm{m}_{i j p t}}}{T_{i j p t}}=\frac{\left(\pi G_{i p} L_{i p}\right) /\left(12 v_{i j p t} f_{i j p t}\right)}{T C_{j} /\left(v_{i j p t}^{\alpha_{j}} f_{i j p t}^{\beta_{j}} d_{i p}^{\gamma_{j}}\right)}=\frac{\pi G_{i p} L_{i p} d_{i p}^{\gamma_{j}}}{12 T C_{j} v_{i j p t}^{\left(1-\alpha_{j}\right)} f_{i j p t}^{\left(1-\beta_{j}\right)}} .
$$

Consequently, $q_{i j p t}=\left\lfloor 1 / U_{i j p t}\right\rfloor$ and $n_{i j p t}=\left\lceil Q_{p t} / q_{i j p t}\right\rceil$. The wear land is the tool life criterion in Taylor's equation although this criterion is not practical in a factory environment because of the difficulties and time required to measure it. Therefore we calculate the workpiece count, $q_{i j p t}$, in order to instruct either the CNC program or the operator to change tools after a predetermined number of pieces have been machined. Furthermore, all time consuming events except the actual machining operation are called non-machining time components. Although there may be other distinct non-machining time components such as tool tuning, workpiece loading/unloading, we consider only the ones that can be expressed as a function of both the machining conditions and alternative operation-tool pairs, such as tool switching times, $r_{j}$. The tool switching time is the time required to replace the worn tool with a new one; each tool type might have a different switching time depending on whether or not the tool uses some special accessory.

A mathematical formulation of the problem can be as follows:

$$
\begin{aligned}
\operatorname{Minimize} & \sum_{p \in \boldsymbol{P}} \sum_{t \in \boldsymbol{T}} S_{p t} Y_{p t}+\sum_{p \in \boldsymbol{P}} \sum_{t \in T} h_{p t} I N_{p t}+\sum_{p \in \boldsymbol{P}} \sum_{t \in \boldsymbol{T}} D_{p t} C_{0} \sum_{i \in I_{p}} \sum_{j \in \sigma_{i}} x_{i j p t} t_{\mathrm{m}_{i j p t}} \\
& +\sum_{p \in \boldsymbol{P}} \sum_{t \in T} C_{0} Q_{p t} \sum_{i \in I_{p}} \sum_{j \in \sigma_{i}} U_{i j p t} r_{j}+\sum_{p \in \boldsymbol{P}} \sum_{t \in T} Q_{p t} \sum_{i \in I_{p}} \sum_{j \in \sigma_{i}} U_{i j p t} C_{t_{j}}
\end{aligned}
$$

Subject to:

Production and inventory balance constraints

$$
\begin{aligned}
& Q_{p t}+I N_{p, t-1}-I N_{p t}=D_{p t}, \quad \text { for every } p \in P, t \in T . \\
& Q_{p t} \leqslant M Y_{p t} \text { for every } p \in P, t \in T .
\end{aligned}
$$

Machine hour availability constraints

$$
\sum_{p \in P} Q_{p t} \sum_{i \in I_{p}} \sum_{j \in \sigma_{i}} x_{i j p t} t_{\mathrm{m}_{i j p t}}+\sum_{p \in P} Q_{p t} \sum_{i \in I_{p}} \sum_{j \in \sigma_{i}} x_{i j p t} U_{i j p t} r_{j}+\sum_{p \in P} t s_{p t} Y_{p t} \leqslant M H_{t} \quad \text { for every } t \in T .
$$

\section{Tool assignment constraints}

$$
\begin{aligned}
& \sum_{j \in \sigma_{i}} x_{i j p t}-Y_{p t}=0, \text { for every } i \in I_{p}, p \in P, t \in T . \\
& x_{i j p t} \geqslant U_{i j p t}, \quad \text { for every } i \in I_{p}, j \in J, p \in P, t \in T .
\end{aligned}
$$




\section{Tool availability constraints}

$$
\sum_{p \in P} \sum_{i \in I_{p}} x_{i j p t} Q_{p t} U_{i j p t} \leqslant N_{j t}, \quad \text { for every } j \in J, t \in T .
$$

\section{Tool life constraints}

$$
x_{i j p t} U_{i j p t} q_{i j p t} \leqslant 1, \text { for every } i \in I_{p}, j \in J, p \in P, t \in T .
$$

Machine power constraints

$$
x_{i j p t} C_{\mathrm{m}} v_{i j p t}^{b} f_{i j p t}^{c} d_{i p}^{e} \leqslant H P, \quad \text { for every } i \in I_{p}, j \in J, p \in P, t \in T .
$$

Surface roughness constraints

$$
x_{i j p t} C_{\mathrm{s}} v_{i j p t}^{g} f_{i j p t}^{h} d_{i p}^{l} \leqslant S F_{i p}, \quad \text { for every } i \in I_{p}, j \in J, p \in P, t \in T .
$$

Non-negativity and integrality constraints

$$
v_{i j p t}, f_{i j p t}>0, \quad Q_{p t}, I_{p t} \geqslant 0, \quad x_{i j p t}, Y_{p t}=\{0,1\}
$$

and $q_{i j p t}$ positive integer for every $p \in P, i \in I_{p}, j \in J, t \in T$

In this non-linear MIP formulation, the objective function is composed of setup, inventory holding, machining, non-machining and tooling costs, respectively. If a tool is not fully utilized for machining a part then it can be used for machining another part, each one with different machining conditions. Therefore we introduce the actual tool usage concept, $U_{i j p t}$. The first set of constraints are production and inventory balance constraints in which both the amount of inventory left in stock at the end of each period and the demand in each period are supplied by either the amount of production in each period or the amount of inventory carried over from the previous period. The second set of constraints ensures that for each period total time required, which is composed of machining, non-machining and setup time components, does not exceed available machine hour. The third set of constraints represents the operational constraints which guarantee that if a certain part is produced in a given period, i.e. $Y_{p t}=1$, then each operation of this part is assigned to a single-tool type of its candidate tools set. We consider a set of alternative cutting tool types for each machining operation, since no one cutting tool type is best for all purposes. The fourth set of constraints ensures that total tool requirement does not exceed the amount of tools on hand. The fifth set of constraints guarantees that machining time for an operation does not exceed available tool life and finally the last two sets of constraints represent usual machining operation constraints. The surface roughness presents the quality requirement on the operation and the machine power constraint ensures that the machining conditions will be set within the limitations of available horsepower.

\section{Algorithms}

The constraints and the decision variables for lot sizing, tool allocation and machining conditions interact with each other. In order to solve the integrated problem, we also assume that if 
a production takes place for part $p$ in a period $t$ then the entering inventory of part $p$ for period $t$ must be zero, i.e. $Q_{p t} I N_{p, t-1}=0$. This assumption is also known as the Wagner-Whitin property, which implies that the lot size $Q_{p t}$ can only assume the values of $0, D_{p t}, D_{p t}+D_{p, t+1}, \ldots$, etc. In this section, we will propose five alternative joint solution algorithms in order to solve these interrelated problems simultaneously. The first algorithm finds the global optimum solution, whereas the other ones are heuristics and guarantee at least local optimality. The underlying reasoning for all of the algorithms is similar such that we first relax the machine hour availability constraint, which can be called a coupling constraint among the parts. As a result, the problem decomposes into several tool management subproblems, one for each part. For the reduced problem, we then relax the set of tool availability constraints. In this resource directed decomposition procedure, we first find the optimum machining conditions for every possible operation-tool pair and select the tool that gives the minimum cost by using the single-machining operation problem (SMOP). This provides a lower bound for tool allocation and machining conditions optimization problem, since we ignore the contention among the different operations for a limited number of tools in addition to the contention among the parts that will be manufactured on the same CNC machine. The main advantage of the proposed decomposition procedure is the computational tractability. As discussed below, we can calculate the optimum machining conditions for every operation-tool pair very quickly to generate a set of alternative solutions, afterwards we impose the relaxed constraints to find the optimum combination of alternatives. Consequently, the non-linear MIP formulation with several set of constraints given in the previous section is polynomially transformed to a much simpler IP formulation.

In SMOP, the objective function includes the operating cost due to the machining, nonmachining and tooling costs subject to the machining and tool life constraints. The following geometric programming (GP) formulation can be written for every possible operation-tool pair for a given lot size:

$$
\begin{array}{ll}
\text { Minimize } & S M O P_{i j p t}=C_{0} t_{m_{i j p t}}+C_{0} r_{j} U_{i j p t}+C_{t_{j}} U_{i j p t}=C_{1} v_{i j p t}^{-1} f_{i j p t}^{-1}+C_{2} v_{i j p t}^{\left(\alpha_{j}-1\right)} f_{i j p t}^{\left(\beta_{j}-1\right)} \\
\text { Subject to } & C_{t}^{\prime} v_{i j p t}^{\left(\alpha_{j}-1\right)} f_{i j p t}^{\left(\beta_{j}-1\right)} \leqslant 1 \quad \text { (Tool life constraint) } \\
& C_{\mathrm{m}}^{\prime} v_{i j p t}^{b} f_{i j p t}^{c} \leqslant 1 \quad \text { (Machine power constraint). } \\
& C_{\mathrm{s}}^{\prime} v_{i j p t}^{g} f_{i j p t}^{h} \leqslant 1 \quad \text { (Surface roughness constraint). } \\
& v_{i j p t}, f_{i j p t}>0
\end{array}
$$

where

$$
\begin{aligned}
& C_{1}=\frac{\pi G_{i p} L_{i p} C_{0}}{12}, \quad C_{2}=\frac{\pi G_{i p} L_{i p} d_{i}^{\gamma_{j}}\left(C_{t_{j}}+C_{0} r_{j}\right)}{12 T C_{j}} \\
& C_{\mathrm{t}}^{\prime}=\frac{\pi G_{i p} L_{i p} d_{i p}^{\gamma_{j}} q_{i j p t}}{12 T C_{j}}, \quad C_{\mathrm{m}}^{\prime}=\frac{C_{\mathrm{m}} d_{i p}^{e}}{H P} \quad \text { and } \quad C_{\mathrm{s}}^{\prime}=\frac{C_{\mathrm{s}} d_{i p}^{l}}{S F_{i p}} .
\end{aligned}
$$

Denoting the dual variables by $Z_{1}, Z_{2}, \ldots, Z_{5}$ the GP-Dual formulation for the above problem is given below. The first two dual variables $Z_{1}$ and $Z_{2}$ correspond to the each of the primal objective function terms, respectively. Hence, their summation must be equal to 1 , also known as normality 
constraint, as stated in the first dual constraint. The other dual variables $Z_{3}, Z_{4}$ and $Z_{5}$ correspond to the primal problem constraints, respectively. Furthermore, there is a dual constraint for each primal variable, $v_{i j p t}$ and $f_{i j p t}$, respectively, known as orthogonality constraints:

$$
\begin{array}{ll}
\text { Maximize } & G D P_{i j p t}=\left(\frac{C_{1}}{Z_{1}}\right)^{Z_{1}}\left(\frac{C_{2}}{Z_{2}}\right)^{Z_{2}}\left(C_{\mathrm{t}}^{\prime}\right)^{Z_{3}}\left(C_{\mathrm{m}}^{\prime}\right)^{Z_{4}}\left(C_{\mathrm{s}}^{\prime}\right)^{Z_{5}} \\
\text { Subject to } & Z_{1}+Z_{2}=1 \\
& -Z_{1}+\left(\alpha_{j}-1\right) Z_{2}+\left(\alpha_{j}-1\right) Z_{3}+b Z_{4}+g Z_{5}=0, \\
& -Z_{1}+\left(\beta_{j}-1\right) Z_{2}+\left(\beta_{j}-1\right) Z_{3}+c Z_{4}+h Z_{5}=0, \\
& Z_{1}, Z_{2}, Z_{3}, Z_{4}, Z_{5} \geqslant 0 .
\end{array}
$$

The objective for the dual problem is still nonlinear, but the constraints of the dual formulation are well-defined linear equations. The dual problem can be solved by using the complementary slackness conditions between dual variables and primal constraints, in addition to constraints of both the primal and dual problems. Each of the constraints of the primal problem can be either loose or tight at optimality. If a dual feasible solution is found for a given problem then the corresponding primal solution can be evaluated in terms of its decision variables, and consequently the primal feasibility of the solution can be checked. At optimality, the corresponding solution should be feasible in both the dual and primal problems, and the objective function value for both problems should be the same. Since we have three constraints in the problem, there are eight different cases for the dual. Let us look at the one of these cases to show how we derived closed-form expressions for primal and dual variables. If only the surface roughness constraint is tight then $Z_{5}$ should be non-negative because of the dual feasibility constraints. Furthermore, the tool life and the machine power constraints are loose, so the corresponding dual variables $Z_{3}$ and $Z_{4}$ are both equal to zero due to the complementary slackness conditions. Therefore, the constraints of GP-dual problem are reduced to the following system:

$$
\begin{aligned}
& Z_{1}+Z_{2}=1, \\
& -Z_{1}+\left(\alpha_{j}-1\right) Z_{2}+g Z_{5}=0, \\
& -Z_{1}+\left(\beta_{j}-1\right) Z_{2}+h Z_{5}=0 .
\end{aligned}
$$

The solution for this system can be stated explicitly as follows:

$$
Z_{1}=1-Z_{2}, \quad Z_{2}=\frac{g-h}{g \beta_{j}-h \alpha_{j}} \text { and } Z_{5}=\frac{\alpha_{j}-\beta_{j}}{h \alpha_{j}-g \beta_{j}},
$$

where $g \beta_{j}-h \alpha_{j} \neq 0$, since $g<0, \alpha_{j}, \beta_{j}>1$ and $h>0$.

The following conditions should be satisfied to verify dual feasibility of the solution:

$$
0 \leqslant Z_{1}, \quad Z_{2} \leqslant 1 \text { and } Z_{5} \geqslant 0 .
$$


Otherwise, this case is infeasible and we continue with another case. If the solution is both primal and dual feasible that means it is optimal then

$$
S M O P_{i j p t}=G P D_{i j p t}=\left(\frac{C_{1}}{Z_{1}}\right)^{Z_{1}}\left(\frac{C_{2}}{Z_{2}}\right)^{Z_{2}}\left(C_{\mathrm{s}}^{\prime}\right)^{Z_{5}}
$$

In the geometric programming, dual variables $Z_{1}$ and $Z_{2}$ give the weight of each term in the primal objective function as discussed in Akturk and Avci [19]:

$$
Z_{2}=\frac{C_{2} v_{i j p t}^{(\alpha-1)} f_{i j p t}^{(\beta-1)}}{S M O P_{i j p t}}=\frac{g-h}{g \beta_{j}-h \alpha_{j}} \quad \text { and } \quad Z_{1}=\frac{C_{1} v_{i j p t}^{-1} f_{i j p t}^{-1}}{S M O P_{i j p t}}=1-Z_{2} .
$$

By taking logarithmic transform, above system turns to a system of linear equations with two equations and two unknowns, which is solved for $v_{i j p t}$ and $f_{i j p t}$, as follows:

$$
\begin{aligned}
f_{i j p t} & =\exp \left(\frac{\ln \left(Z_{2} S M O P_{i j p t} / C_{2}\right)+\left(\alpha_{j}-1\right) \ln \left(Z_{1} S M O P_{i j p t} / C_{1}\right)}{\beta_{j}-\alpha_{j}}\right), \\
v_{i j p t} & =\frac{C_{1}}{f_{i j p t} Z_{1} S M O P_{i j p t}} .
\end{aligned}
$$

After finding $v_{i j p t}$ and $f_{i j p t}$, we can check the feasibility of tool life and machine power constraints. As demonstrated on this case, we can find the solution of SMOP very quickly since the explicit analytic expressions of the solution exist for all cases.

In all of the proposed algorithms, namely exact, look ahead and single pass, we first determine all alternative lot sizes. An alternative $k$ for a period $t$ means that production in period $t$ satisfies cumulative demand of $k$ periods including period $t$. Therefore $k$ is in the range of $[1, \ldots,|T|-t+1]$ for any period $t$, where $|T|$ denotes the cardinality of the set $T$. Afterwards we solve tool allocation and machining conditions optimization problem for a given lot size to calculate total cost and machine hour requirements for alternative lot sizes. In the exact algorithm, we finally construct and solve an IP formulation to find the optimum solution. In the second and the third algorithms, we reduce the search space using either least unit cost (LUC) or least period cost (LPC) as a stopping criterion, which in turn decreases the size of the IP formulation in final step of these algorithms. In these algorithms, we determine all possible lot sizes similar to the exact algorithm, although we stop solving the tool allocation and machining conditions optimization problem when we reach a local minimum for cost per unit or cost per period measures. Therefore we may deviate from global optimum due to the differences in capacity levels between periods, but gain considerably from computation time. The last two algorithms are similar to the second and third algorithms, however in these algorithms unlike the previous ones we present the alternatives with minimum cost per unit and cost per period values as the final solution without solving an IP formulation. A step-by-step execution of all of these algorithms is given on a numerical example in Section 4.

The following additional notation is used in the proposed algorithms:

$C_{p t k}$ total cost for alternative $k$ of part $p$ in period $t(\$)$

$K_{p t}$ set of feasible alternatives of part $p$ in period $t$ 
$Q_{p t k}$ lot size for alternative $k$ of part $p$ in period $t$

$R_{j p t k}$ tool type $j$ requirement for alternative $k$ of part $p$ in period $t$

$T_{p t k}$ total machine hour requirement for alternative $k$ of part $p$ in period $t(\mathrm{~min})$

$z_{p t k} 0-1$ binary decision variable which is equal to 1 , if alternative $k$ is selected for part $p$ in period $t$

\subsection{Exact algorithm}

The steps of the proposed exact algorithm can be summarized as follows. In the first step we determine alternative lot size values for all parts and periods. In step 2, we determine the optimum tool allocations and machining conditions for each alternative lot size. For example, in step 2.1, we initially set $q_{i j p t}=\left\lceil Q_{p t k} / N_{j t}\right\rceil$, i.e. tool availability constraints are relaxed, and find the optimum machining conditions $v_{i j p t}, f_{i j p t}$ and $U_{i j p t}$ for every operation-tool pair $(i, j)$ of part $p$. If this solution violates the tool availability constraints, that means the required number of tools for a tool type $j$ is greater than the amount of tools on hand for a given lot size alternative $Q_{p t k}$, then we generate a set of alternative tool allocations denoted as $b_{i j p t}^{m}$ in step 2.2. Consequently, for every $(i, j)$ pair of part $p$, we evaluate each tool allocation alternative $m \in\left\{1,2, \ldots, n_{i j p t}\right\}$, where $n_{i j p t}$ is the required number of tools for the relaxed problem as stated in step 2.1. We now set $q_{i j p t}=\left\lceil Q_{p t k} / m\right\rceil$, i.e. $m<N_{j t}$, and resolve SMOP for each value of $m$, hence $S M O P_{i j p t}^{m}$ is the optimum objective function value of the SMOP function at the tool requirement level $m$. For example, $m=1$ means only a single tool of type $j$ is assigned to complete operation $i$ of part $p$ for a given lot size alternative $Q_{p t k}$. Therefore, we have to decrease either the cutting speed $v_{i j p t}$ or the feed rate $f_{i j p t}$, or both, which corresponds to increasing machine time and also tool life, to increase the usage rate $U_{i j p t}$, so that more parts can be machined for a given tool life. When the while loop in step 2.3 is executed for a lot $k$ of a certain $(p, t)$ pair, the tool management algorithm may not be able to find a feasible solution due to the insufficient machine capacity. In this case, we do not need to check for larger lot sizes and exit the while loop in order to continue with another $(p, t)$ pair. Consequently, different lot sizing alternatives might lead to different machining conditions and tool allocations. Therefore, in the third step, we compute total cost, machine hour and tool requirements for all feasible alternatives. Hence one alternative might require more machining time $T_{p t k}$, but less number of tools $R_{j p t k}$, whereas another alternative might give a less machining time in albeit of higher cost $C_{p t k}$. Finally in step 4, we solve an IP formulation to find the optimum combination of alternatives.

Step 1: (Determination of possible lot sizes) For every $p \in P$ and $t \in T$. Set $k=1$. While $(k \leqslant|T|-t+1) Q_{p t k}=\sum_{r=t}^{t+k-1} D_{p r}$ and $k=k+1$.

Step 2: (Tool allocation and machining conditions optimization) For every $p \in P$ and $t \in T$, set $k=1$, $K_{p t}=\emptyset$ and do the following while $(k \leqslant|T|-t+1)$.

Step 2.1: For every possible $(i, j)$ pair, initially set $q_{i j p t}=\left\lceil Q_{p t k} / N_{j t}\right\rceil$ and solve SMOP to determine optimum $v_{i j p t}, f_{i j p t}$ and $U_{i j p t}$. Consequently, $q_{i j p t}=\left\lfloor 1 / U_{i j p t}\right\rfloor$ and $n_{i j p t}=\left\lceil Q_{p t k} / q_{i j p t}\right\rceil$.

Step 2.2: If $\sum_{i \in I_{p}} \sum_{p \in P} U_{i j p t} Q_{p t k} \leqslant N_{j t}$ for every $j \in J$ in a given period $t$ then the lower bound solution found in Step 2.1 gives the optimum tool allocations and machining conditions, go to Step 2.3. Otherwise resolve SMOP for the requirement level, $m \in\left\{1,2, \ldots, n_{i j p t}\right\}$, hence $q_{i j p t}=\left\lceil Q_{p t k} / m\right\rceil$, of every operation $(i, j)$ to find $v_{i j p t}^{m}, f_{i j p t}^{m}, U_{i j p t}^{m}$, and the corresponding $S M O P_{i j p t}^{m}$. Solve the following integer programming (IP) formulation to find the best allocation for every operation that 
satisfies the tool availability constraints in a given period $t$ :

$$
\begin{array}{ll}
\text { Minimize } & \sum_{p \in P} \sum_{i \in I_{p}} \sum_{j \in \sigma_{i}} \sum_{m=1}^{n_{i j p t}} Q_{p t k} S M O P_{i j p t}^{m} b_{i j p t}^{m} \\
\text { Subject to } & \sum_{j \in \sigma_{i}} \sum_{p \in P} \sum_{m=1}^{n_{i j p t}} b_{i j p t}^{m}=1, \quad \forall i \in I_{p}, \\
& \sum_{i \in I_{p}} \sum_{p \in P} \sum_{m=1}^{n_{i j p t}} m b_{i j p t}^{m} \leqslant N_{j t}, \quad \forall j \in J,
\end{array}
$$

where $b_{i j p t}^{m}$ is a $0-1$ binary decision variable which is equal to 1 if the operation $i$ of part $p$ is assigned to tool $j$ in period $t$ at the tool requirement level of $m$ tools. The first set of constraints, also considering the alternative tools, ensures that a single allocation will be selected for each operation. The second set of constraints guarantees that total number of tool allocations will not exceed the tool availability constraints.

Step 2.3: If there is a feasible solution for a given $Q_{p t k}$ then set $K_{p t}=K_{p t} \cup\{k\}, k=k+1$. Else exit this loop to continue with another $(p, t)$ pair.

Step 3: (Determination of total cost, machine hour and tool requirements) For every $p \in P, t \in T$ and $k \in K_{p t}$ find $C_{p t k}, T_{p t k}$ and $R_{j p t k}$ as follows, where $*$ denotes the optimum value:

$$
\begin{aligned}
& -C_{p t k}=Q_{p t k} \sum_{i \in I_{p}} \sum_{j \in \sigma_{i}} S M O P_{i j p t}^{*}+S_{p t}+\sum_{s=t+1}^{t+k-1} D_{p s} \sum_{r=t}^{s-1} h_{p r}, \\
& -T_{p t k}=Q_{p t k} \sum_{i \in I_{p}}\left(\frac{\pi G_{i p} L_{i p}}{12 v_{i j p t}^{*} f_{i j p t}^{*}}+U_{i j p t}^{*} r_{j}\right)+t s_{p t}, \\
& -R_{j p t k}=Q_{p t k} \sum_{i \in I_{p}} U_{i j p t}^{*} \quad \text { for every } j \in J .
\end{aligned}
$$

Step 4: Solve the following 0-1 IP formulation to find the optimum combination of alternatives.

$$
\begin{array}{ll}
\text { Minimize } & \sum_{p \in P} \sum_{t \in T} \sum_{k \in K_{p t}} C_{p t k} z_{p t k} \\
\text { Subject to } & \sum_{k \in K_{p 1}} z_{p 1 k}=1 \quad \text { for every } p \in P . \\
& \sum_{k \in K_{p t}} z_{p t k}-\sum_{r=1}^{t-1} z_{p, r, t-r} \geqslant 0 \quad \text { for every } p \in P, t=2, \ldots,|T| . \\
& \sum_{p \in P} \sum_{k \in K_{p t}} R_{j p t k} z_{p t k} \leqslant N_{j t} \quad \text { for every } t \in T, j \in J . \\
& \sum_{p \in P} \sum_{k \in K_{p t}} T_{p t k} z_{p t k} \leqslant M H_{t} \quad \text { for every } t \in T . \\
& z_{p t k} \in\{0,1\} \quad \text { for every } p \in P, \quad t \in T, k \in K_{p t} .
\end{array}
$$

In the above formulation the first set of constraints ensures that for each part $p$ exactly one alternative is selected for period 1, since we do not allow backlogging. By the second set of 
constraints, it is guaranteed that for each part demand is satisfied and finally the last two sets of constraints ensure that tool and machine hour availability limits are not exceeded, respectively. In this IP formulation, the number of integer variables and the number of constraints are bounded by $P T(T+1) / 2$ and $T(1+P+J)+P$, respectively.

\subsection{Look-ahead algorithms}

In the look-ahead algorithms, we determine all alternative lot sizes similar to the previous algorithm, but we try to decrease the number of $z_{p t k}$ variables to reduce the size of the IP formulation given in step 4. Hence steps 1, 2.1, 2.2, 3 and 4 are same as the exact algorithm. However in step 2.3 we generate alternative lot sizes $Q_{p t k}$ and their corresponding optimum tool allocations and machining conditions until we reach a local optimum either for least unit cost (LUC) or for least period cost (LPC) criterion, denoted as LA-LUC and LA-LPC, respectively. The LPC method selects the lot size such a way that the total relevant costs per unit time for the duration of the lot size quantity are minimized. For example, for period 1, a lot size for part $p$ that covers requirements out to period $t$ is $Q_{p 1 t}=D_{p 1}+D_{p 2}+\cdots+D_{p t}$. The setup cost for this lot is $S_{p 1}$ and its holding cost $H_{p 1 t}=H_{p 2} D_{p 2}+2 h_{p 3} D_{p 3}+\cdots+(t-1) h_{p t} D_{p t}$. Consequently, the total relevant cost is $C_{p 1 t}=S_{p 1}+H_{p 1 t}$, thus its total relevant cost per unit time is $C_{p 1 t} / t$. The LUC method is similar to the LPC method except with a different cost criterion such that it minimizes the total relevant cost per unit, i.e. $C_{p 1 t} / Q_{p 1 t}$. Consequently, we get a reduced set of alternative lot sizes at the end of the first two steps, and solve an IP formulation to find the optimum combination of available alternatives. Therefore, the new step 2.3 can be written for the LA-LUC algorithm as follows:

Step 2.3: For every $p \in P$ and $t \in T$, set $k=1, K_{p t}=\emptyset$ and $L U C=\infty$.

While $(k \leqslant|T|-t+1)$

- Calculate the tool management decisions for a given lot size $Q_{p t k}$ and compute $C_{p t k}$ and $T_{p t k}$ as in step 3 of the exact algorithm.

- If $C_{p t k} / Q_{p t k}<L U C$ then set $L U C=C_{p t k} / Q_{p t k}, K_{p t}=K_{p t} \cup\{k\}$, and $k=k+1$.

- Else exit this loop to continue with another $(p, t)$ pair.

For the LA-LPC algorithm, the only difference is to use LPC instead of LUC as a criterion to reduce the search space such that if $C_{p t k} / k<L P C$ then set $L P C=C_{p t k} / k, K_{p t}=K_{p t} \cup\{k\}$ and $k=k+1$. Else exit this loop to continue with another $(p, t)$ pair.

\subsection{Single-pass algorithms}

In the single-pass algorithms, instead of solving an IP formulation as in the exact and look-ahead algorithms, we find an alternative with either the minimum least unit cost or the least period cost, denoted as SP-LUC and SP-LPC respectively, as in the look ahead algorithms. Therefore the final solution for the SP-LUC algorithm is found in step 3 as follows:

Step 3: For every $p \in P$, set $t=1$.

While $(t \leqslant|T|)$, find $r=\operatorname{argmin}_{k \in K_{p t}}\left\{C_{p t k} / Q_{p t k}\right\}$ and set $z_{p t r}=1$ and $t=t+r$.

Whereas in the SP-LPC algorithm, the final solution is found with respect to the number of periods instead of the number of units as follows:

While $(t \leqslant|T|)$, find $r=\operatorname{argmin}_{k \in K_{p t}}\left\{C_{p t k} / t\right\}$ and set $z_{p t r}=1$ and $t=t+r$. 


\section{Numerical example}

In this section, we will discuss the detailed execution of all of the algorithms over an example problem. In this example, there are five parts which require six tool types with different technological data. The detailed data is presented for part 1 only as an example due to space limitations in Tables 1-3. Machine hour availabilities for periods 1-8 are (2600, 2300, 2400, 2400, 2500, 2500, 2400, 2200), respectively.

In the first step of the exact algorithm, we find the possible lot sizes for all parts and periods. In step 2, we determine optimum machining conditions and tool allocations for any period of any part as long as a feasible solution is found. In the next step, we determine total cost and machine hour values for the lot sizes for which tool management algorithm gives a feasible solution. The possible lot sizes $\left(Q_{p t k}\right)$ and corresponding cost $\left(C_{p t k}\right)$ values for part 1 obtained at the end of first three steps are presented in Table 4. Finally, we solve an IP formulation to find the optimum combination of alternatives in step 4.

In the look ahead algorithms, LA-LUC and LA-LPC, we determine possible lot sizes as in the exact algorithm, however we stop solving the tool management algorithm when we reach a local optimum for cost per unit and cost per period measures, respectively. As an example, in the LA-LUC algorithm for $p=1$ and $t=1$, since $C_{111} / Q_{111}=78.6 / 40=1.9$ and $C_{112} / Q_{112}=$ $149.6 / 65=2.3$, we no longer iterate in step 2.3 and consequently, $K_{11}$ contains only the first alternative. Similarly, in LA-LPC algorithm for $p=1$ and $t=1$, since $C_{111} / 1=78.6 / 1=$ $78.6, C_{112} / 2=149.6 / 2=74.8$ and $C_{113} / 3=264.2 / 3=88.1, K_{11}$ contains only the first and the

Table 1

Cost and time data for part 1

\begin{tabular}{|c|c|c|c|c|c|c|c|c|}
\hline & \multicolumn{8}{|c|}{ Period no. $(t)$} \\
\hline & 1 & 2 & 3 & 4 & 5 & 6 & 7 & 8 \\
\hline$D_{p t}$ & 40 & 25 & 30 & 30 & 30 & 20 & 40 & 45 \\
\hline$S_{p t}$ & 10.3 & 10.9 & 11.0 & 11.0 & 9.1 & 10.2 & 8.7 & 8.2 \\
\hline$t s_{p t}$ & 10.2 & 8.7 & 7.5 & 9.3 & 11.1 & 10.5 & 6.3 & 9.3 \\
\hline$h_{p t}$ & 0.14 & 0.15 & 0.15 & 0.15 & 0.12 & 0.14 & 0.12 & 0.11 \\
\hline
\end{tabular}

Table 2

Operation-tool assignments and operation data for part 1

\begin{tabular}{llllllllll}
\hline Operation no. $(i)$ & \multicolumn{1}{l}{ Tool no. $(j)$} & & & & $S F_{i p}$ & $d_{i p}$ & $G_{i p}$ & $L_{i p}$ \\
\hline 1 & 1 & 0 & 0 & 1 & 1 & 0 & 89 & 0.04 & 2.0 \\
2 & 0 & 1 & 0 & 0 & 1 & 1 & 451 & 0.10 & 1.7 \\
3 & 1 & 0 & 0 & 0 & 0 & 1 & 427 & 0.20 & 3.1 \\
4 & 0 & 0 & 0 & 0 & 1 & 0 & 45 & 0.10 & 2.1 \\
5 & 1 & 1 & 0 & 0 & 0 & 1 & 204 & 0.11 & 3.2 \\
\hline
\end{tabular}


Table 3

Tool availability and tooling information

\begin{tabular}{|c|c|c|c|c|c|c|c|c|c|c|}
\hline \multirow[t]{2}{*}{ Tool no. $(j)$} & \multicolumn{8}{|c|}{ Period no. $(t)$} & \multirow[t]{2}{*}{$C_{t_{j}}$} & \multirow[t]{2}{*}{$r_{j}$} \\
\hline & 1 & 2 & 3 & 4 & 5 & 6 & 7 & 8 & & \\
\hline 1 & 8 & 10 & 10 & 10 & 12 & 9 & 10 & 7 & 3.52 & 1.44 \\
\hline 2 & 11 & 12 & 8 & 10 & 12 & 11 & 8 & 10 & 3.28 & 1.20 \\
\hline 3 & 12 & 10 & 10 & 12 & 12 & 11 & 10 & 8 & 3.87 & 1.14 \\
\hline 4 & 10 & 11 & 10 & 11 & 7 & 7 & 11 & 9 & 3.85 & 1.34 \\
\hline 5 & 7 & 9 & 12 & 9 & 11 & 10 & 9 & 9 & 3.15 & 1.27 \\
\hline 6 & 11 & 12 & 12 & 9 & 10 & 8 & 9 & 12 & 3.51 & 1.16 \\
\hline
\end{tabular}

Table 4

Alternative lot sizes and total cost values $\left(Q_{p t k} / C_{p t k}\right)$ for part 1

\begin{tabular}{|c|c|c|c|c|c|c|c|c|}
\hline \multirow[t]{2}{*}{$t$} & \multicolumn{8}{|c|}{ Alternative no. $(k)$} \\
\hline & 1 & 2 & 3 & 4 & 5 & 6 & 7 & 8 \\
\hline 1 & $40 / 78.6$ & $65 / 149.6$ & $95 / 264.2$ & $125 / 412.2$ & $155 / 590.8$ & $175 / 726.4$ & $215 / 1033.5$ & 260/Inf. \\
\hline 2 & $25 / 51.3$ & $55 / 152.1$ & $85 / 294.5$ & $115 / 483.4$ & $135 / 632.4$ & $175 / 985.5$ & 220/Inf. & \\
\hline 3 & $30 / 58.5$ & $60 / 133.6$ & $90 / 238.6$ & $110 / 327.1$ & $150 / 541.7$ & $195 / 841.0$ & & \\
\hline 4 & $30 / 56.4$ & $60 / 136.4$ & $80 / 208.9$ & $120 / 400.5$ & $165 / 682.9$ & & & \\
\hline 5 & $30 / 62.2$ & $50 / 129.1$ & $90 / 324.0$ & $135 / 639.3$ & & & & \\
\hline 6 & $20 / 38.5$ & $60 / 133.8$ & $105 / 301.0$ & & & & & \\
\hline 7 & $40 / 75.4$ & $85 / 211.3$ & & & & & & \\
\hline 8 & $45 / 105.7$ & & & & & & & \\
\hline
\end{tabular}

second alternatives. In these algorithms, finally we solve an IP formulation to find the best combination of available alternatives.

In the single-pass algorithms, SP-LUC and SP-LPC, we again determine lot sizes and solve tool management problem as described above. However, in these algorithms we do not solve the final IP formulation, instead we present the alternatives with minimum cost per unit and cost per period measures as the final solution. Thus, for $p=1$ and $t=1$ the proposed lot sizes for SP-LUC and SP-LPC are $Q_{111}=40$ and $Q_{112}=65$, respectively.

We also solved this numerical example using three well-known uncapacitated lot sizing algorithms used in MRP II packages, namely Wagner-Whitin (WW), least unit cost (LUC) and least period cost (LPC), by utilizing a two-level approach. In the first level, we found the lot sizes that minimized the sum of setup and inventory holding costs. An optimum tool allocation and machining conditions were calculated for the given lot sizes in the second level. For this numerical example, the total cost values found by the two-level methods and the proposed algorithms along with the percent improvements are given in Table 5. In this table, percent improvement 
Table 5

Total cost values and percent improvements

\begin{tabular}{lllllll}
\hline & & Exact & LA-LUC & LA-LPC & SP-LUC & SP-LPC \\
& Cost & 2098.1 & 2099.0 & 2108.7 & 2188.2 \\
\hline WW & 2590.2 & $23.4 \%$ & $23.4 \%$ & $22.8 \%$ & $23.1 \%$ & $18.4 \%$ \\
LUC & 2550.6 & $21.6 \%$ & $21.5 \%$ & $20.9 \%$ & $21.2 \%$ & $16.6 \%$ \\
LPC & 2585.7 & $23.2 \%$ & $23.2 \%$ & $22.6 \%$ & $22.9 \%$ & $18.2 \%$ \\
\hline
\end{tabular}

$=100(T C-J C) / J C$ where $J C$ and $T C$ denote the total cost values found by using joint and two-level methods, respectively.

\section{Computational results}

The proposed algorithms were coded in C language and the IP formulations were solved by using callable library routines of CPLEX version 5.0 MIP solver on a Sparc station 10 under SunOS 5.4. In this section, the efficiency of the proposed joint algorithms were tested by comparing the total costs found by these algorithms with the costs found by using two-level WW, LUC and LPC algorithms. There are seven experimental factors that can affect the efficiency of our algorithms, which are listed in Table 6. Both the number of parts and demand rate as well as demand variability are likely to affect the computation times and production costs, where UN stands for the uniform distribution. The setup cost, to make the results of the research meaningful, must be compared to the inventory holding cost as a ratio, $S / I$, as suggested by several authors. The fourth factor is taken as $S / I$ ratio such that the setup cost for each part is equal to the $S / I$ ratio times the inventory holding cost. The fifth and sixth factors specify the cutting tool cost for each tool type and the tightness of the tool availability constraints, respectively. Tightness of the tool availability is likely to affect both computation times and production costs, since if the tool availability constraint is violated for any tool type then we solve an IP formulation to determine the optimum tool allocations and machining conditions in step 2.2. The seventh factor determines the assignment matrix, i.e. random or clustered. At the random level, each cutting tool type can be assigned to a candidate tool set of each operation with an equal probability. In the clustered case the last operation of each part is taken to be finishing operation whereas the remaining operations to be roughing operations. Therefore, each tool is classified as either roughing or finishing tool. As a result, the operation-tool assignment matrix has two disjoint clusters of operation-tool assignments. Since there are seven factors and two levels, our experiment is $2^{7}$ full-factorial design corresponding to 128 combinations. The number of replications for each combination is taken as 5 , giving 640 different randomly generated runs.

Other variables were treated as fixed parameters and generated as follows:

- System related parameters, $C_{0}=\$ 0.5 / \mathrm{min}, H P=5$ h.p.

- Operation related parameters, $G_{i p}$ and $L_{i p}$ were selected randomly from the interval UN $[1.5,2.0]$ and $\mathrm{UN} \sim[2.5,3.0]$, respectively. 
Table 6

Experimental factors

\begin{tabular}{llll}
\hline Factors & Definition & Low & High \\
\hline $\mathrm{A}$ & Number of parts & 10 & 30 \\
$\mathrm{~B}$ & Demand mean $(\mu)$ & 10 & 30 \\
$\mathrm{C}$ & Demand variability & $\mathrm{UN} \sim[0.9 \mu, 1.1 \mu]$ & $\mathrm{UN} \sim[0.6 \mu, 1.4 \mu]$ \\
$\mathrm{D}$ & S/I ratio & 2 & 6 \\
$\mathrm{E}$ & Tooling cost & $\mathrm{UN} \sim[3,4]$ & $\mathrm{UN} \sim[9,10]$ \\
$\mathrm{F}$ & Tool availability & Tight & Loose \\
$\mathrm{G}$ & Assignment matrix & Random & Clustered \\
\hline
\end{tabular}

- Number of operations per part $\mathrm{UN} \sim[3,5]$.

- There were 12 two-shift weekly periods, i.e. $|T|=12$, and $M H_{t}=\mathrm{UN} \sim[4600,5000]$.

- Tool availability for each tool type was taken as a function of the factors A and B, namely part number and demand mean. In low cases of these two factors tool availability was $\mathrm{UN} \sim[2,3]$ and $\mathrm{UN} \sim[10,15]$ for tight and loose cases, respectively. In high cases of these two factors tool availability was $\mathrm{UN} \sim[9,12]$ and $\mathrm{UN} \sim[45,60]$ for tight and loose cases, respectively. Whenever one of these factors was in low and the other was in high case, tool availability was $\mathrm{UN} \sim[4,5]$ and $\mathrm{UN} \sim[20,25]$ for tight and loose cases, respectively.

- The values of $S F_{i p}$ and $d_{i p}$ were related with the assignment matrix. For random assignment matrix, $S F_{i p}=\mathrm{UN} \sim[30,500]$ and $d_{i p}=\mathrm{UN} \sim[0.025,0.3]$. In the clustered case, there were two types of operations, namely roughing and finishing. For roughing operations, $S F_{i p}=\mathrm{UN} \sim[300,500]$ and $d_{i p}=\mathrm{UN} \sim[0.2,0.3]$, and for the finishing operation, $S F_{i p}=\mathrm{UN} \sim[30,70]$ and $d_{i p}=\mathrm{UN} \sim[0.025,0.075]$.

- There were 10 different cutting tool types and $r_{j}=\mathrm{UN} \sim[1,1.5]$.

- The weekly inventory holding cost for each part in each period, $h_{p t}$, was selected randomly from the interval $\mathrm{UN} \sim[0.06,0.08]$. Furthermore, the setup time, $t s_{p t}=(S / I$ ratio $) \cdot \mathrm{UN} \sim[2,3]$ and setup cost, $S_{p t}=(S / I$ ratio $) 50 h_{p t}$, where the constant 50 was used to convert weekly inventory holding costs into yearly equivalents.

In two-level approaches such as WW, LUC and LPC, the lot sizing decision is given without considering its impact on the tool management problem, which can lead to infeasible or inferior results when we consider both the constraints and parameters of the tool management problem. In Table 7, we presented the number of infeasible cases that we encountered in the experimental design out of 640 runs along with the percent improvements achieved over the cases for which the two-level approaches found a feasible solution. Although there is a possibility of infeasibility for the two single-pass algorithms, in our experimental design we did not encounter any such cases. The computation time results for our joint algorithms are presented in Table 8. The proposed joint algorithms dominate and give much better results than any fixed lot size approach due to the increased solution flexibility even though the computational price of the two-level approach is less than the proposed joint approach. Baker [1] has stated that the LPC algorithm is better than the LUC algorithm for the lot sizing problem, however our computational experiments indicate that 
Table 7

Number of infeasible cases and percent improvements

\begin{tabular}{lllllll}
\hline & Inf. cases & Exact & LA-LUC & LA-LPC & SP-LUC & SP-LPC \\
\hline WW & 30 & $6.9 \%$ & $6.5 \%$ & $5.9 \%$ & $5.8 \%$ & $4.7 \%$ \\
LUC & 38 & $7.7 \%$ & $7.2 \%$ & $6.6 \%$ & $6.5 \%$ & $5.4 \%$ \\
LPC & 40 & $7.9 \%$ & $7.5 \%$ & $6.8 \%$ & $6.7 \%$ & $5.6 \%$ \\
\hline
\end{tabular}

Table 8

Computation time (s) results for the joint algorithms

\begin{tabular}{lrrrrr}
\hline & Exact & LA-LUC & LA-LPC & SP-LUC & \multicolumn{1}{c}{ SP-LPC } \\
\hline Min. & 21.5 & 1.2 & 1.2 & 1.0 & 1.0 \\
Ave. & 117.2 & 54.9 & 53.7 & 54.3 & 53.1 \\
Max. & 287.6 & 190.7 & 184.8 & 189.3 & 183.5 \\
\hline
\end{tabular}

the LUC-based algorithms perform better than the LPC-based algorithms in machining environments for both joint and two-level methods since parts might have different processing times and quality requirements.

The statistical analysis of cost, time and improvement data obtained from experimental design has shown that there is a $99 \%$ correlation among our algorithms. Also, it was observed that on the average WW algorithm gives better solutions compared to LUC and LPC algorithms. Therefore for further statistical analysis we used the exact and WW algorithms as representatives of joint and two level approaches. Among the 610 comparable cases, the maximum improvement occurred for the case $\left(\begin{array}{llllll}0 & 1 & 0 & 1 & 0 & 0\end{array}\right)$, where 0 and 1 correspond to the low and high levels of each factor, respectively. We applied a paired-t test to the total cost terms found by these two algorithms to check the statistical significance of their difference. We found that $t$-value was 5.80 and the cost values were different with $p \leqslant 0.000$ significance. As it can be seen from Table 7, WW algorithm resulted in 30 infeasible cases, however these cases were the ones that would increase the average improvement of the exact algorithm over WW algorithm beyond $6.9 \%$ if the $\mathrm{WW}$ algorithm had found comparable feasible results. This fact can be observed in Table 9, where we presented the number of infeasible cases and minimum, average and maximum improvement percentages for the most significant two factors, namely demand mean and $S / I$ ratio, on the percent improvements. It is important to note that in Table 9 since we used the WW algorithm to solve the dynamic lot sizing problem at the first level of the two level approach, we solved both of the levels optimally, but in a top-down hierarchical manner. Therefore, the percent improvements also indicate the average gap from the global optimum solution of the joint problem even if we solve each of these two problems optimally in a two level approach. Although the magnitude of percent improvements depend on the experimental factors as shown in Table 9, we can easily claim that they are significant enough to justify that the proposed algorithms make a strong contribution. 
Table 9

Percent improvements and the number of infeasible cases

\begin{tabular}{llll}
\hline & \multicolumn{2}{l}{$S / I$ ratio } & \\
\cline { 3 - 4 } & & Low (Min., Avg., Max.) & High (Min., Avg., Max.) \\
\hline \multirow{3}{*}{ Average demand } & Low & $(0.70,1.58,3.20)$ & $(0.40,5.38,14.20)$ \\
& No infeasible cases & No infeasible cases \\
& High & $(0.10,7.25,21.50)$ & $(5.80,14.95,36.5)$ \\
& & No infeasible cases & 30 infeasible cases \\
\hline
\end{tabular}

Table 10

$F$ values and significance levels $(p)$ for ANOVA results

\begin{tabular}{|c|c|c|c|c|c|c|}
\hline \multirow[t]{2}{*}{ Factors } & \multicolumn{2}{|c|}{ Total cost } & \multicolumn{2}{|c|}{ Comp. time } & \multicolumn{2}{|c|}{ Improvement } \\
\hline & $F$ & $p$ & $F$ & $p$ & $F$ & $p$ \\
\hline$A$ & 49278.4 & 0.000 & 10580.1 & 0.000 & 6.2 & 0.013 \\
\hline$B$ & 39093.3 & 0.000 & 6.6 & 0.010 & 705.1 & 0.000 \\
\hline C & 0.0 & 0.990 & 1.1 & 0.287 & 7.4 & 0.007 \\
\hline$D$ & 1601.3 & 0.000 & 0.0 & 0.910 & 425.1 & 0.000 \\
\hline$E$ & 1613.3 & 0.000 & 58.8 & 0.000 & 495.1 & 0.000 \\
\hline$F$ & 51.2 & 0.000 & 44.2 & 0.000 & 131.0 & 0.000 \\
\hline$G$ & 181.7 & 0.000 & 1029.0 & 0.000 & 1.8 & 0.177 \\
\hline
\end{tabular}

We also applied a two-way analysis of variance (ANOVA) test on the performance measures of total cost, computation time and percent improvements. The significance levels $(p)$ and $F$ values for these performance measures against seven factors were given in Table 10. As it was expected, all of the factors except the third one, demand variability, were significant for the total production cost with $p \leqslant 0.000$. Among these factors $A$ and $B$ directly affect the amount to be produced, hence total cost of production whereas the fourth and fifth factors affect the setup and tooling cost components of the total production cost, respectively. The sixth factor, tightness of the tool availability, affects the structure of production cost function and hence the total production cost. Finally, the seventh factor affects the total cost of production due to the tool allocation and consequently machining conditions decisions. The ANOVA results for the computation time of the exact algorithm has shown that the most important factors on computation times were the factors $A, E, F$ and $G$ with $p \leqslant 0.000$ significance and the factor $B$ with $p \leqslant 0.010$. Factors $A$ and $B$ directly affect the size of the problem, whereas the factor $F$ constrains the number of tools on hand. The significance of factor $G$, assignment matrix, depends on the fact that, in the clustered case the tool allocation and machining conditions optimization problem is decomposed into two separate problems for roughing and finishing operations, which reduces the number of possibilities. Almost all of the factors were statistically significant on the percent improvements, which also indicated the advantage of the exact algorithm and hence the other proposed joint algorithms, over the two level WW, LUC and LPC algorithms. 


\section{Conclusions}

In this study we have shown that there is a close relationship between the lot sizing and tool management decisions. Therefore, these problems cannot be viewed in isolation. However most of the lot sizing and tool management approaches solve these two problems independently using a two-level approach. In the two-level approach, lot sizing decisions are determined prior to the tool management decisions, which unnecessarily restrict the feasible solution space for the tool management problem, consequently leading to either infeasible or inferior results. We have proposed five solution procedures for the joint problem. The first one is an exact algorithm which guarantees the global optimality. The second and third ones are equipped with a look ahead mechanism to guarantee at least local optimality. As it can be seen from the previous section that the least unit cost criterion gives better results compared to least period cost criterion in such tool management problems both in joint and two level approaches. The proposed algorithms can be used in MRP II softwares to determine lot sizes in conjunction with the tool allocation and machining conditions decisions for a capacitated problem. There are two advantages of the proposed algorithms over the traditional two level approaches. First of all, we guarantee that the lot sizing decisions will satisfy the tool management related constraints so that we ensure overall feasibility. Furthermore, we improve the total production cost by $6.9 \%$ on the average compared to a two level approach which uses the WW algorithm to calculate the lot sizes. The magnitude of cost savings is dependent on the system parameters. At the end, there are several future research directions emanating from this study such as a backlogging option may be incorporated to all of the algorithms. Furthermore, the integrality property assumption may be relaxed to enlarge the solution space by generating different lot sizing alternatives. Finally, we considered only a single CNC machine, however the scope of the study can be extended by considering multiple CNC machines as well as material and tool handling systems.

\section{References}

[1] Baker KR. Requirements planning. In: Graves SC, Rinnooy Kan AHG, Zipkin PH, editors. Handbooks in OR \& MS, vol. 4, 1993. p. 571-627. [Chapter 11].

[2] Kimms A. A genetic algorithm for multi-level, multi-machine lot sizing and scheduling. Computers \& Operations Research 1999;26:829-48.

[3] Stecke KE. Formulation and solution of nonlinear integer production planning problems for flexible manufacturing systems. Management Science 1983;29:273-88.

[4] Gray AE, Seidmann AS, Stecke KE. A synthesis of decision models for tool management in automated manufacturing. Management Science 1993;39:549-67.

[5] Kouvelis P. An optimal tool selection procedure for the initial design phase of a flexible manufacturing system. European Journal of Operational Research 1991;55:201-10.

[6] Wagner HM, Whitin T. Dynamic version of the economic lot size model. Management Science 1958;5:89-96.

[7] Bitran GB, Yanasse HH. Computational complexity of the capacitated lot size problem. Management Science 1982;28:1174-85.

[8] Kirca O, Kokten M. A new heuristic approach for the multi item dynamic lot sizing problem. European Journal of Operational Research 1994;75:332-41.

[9] Thizy JM, Van Wassenhove LN. Lagrangean relaxation for the multi item capacitated lot sizing problem: A heuristic implementation. IIE Transactions 1985;17:308-13. 
[10] Trigeiro WW. A dual cost heuristic for the capacitated lot sizing problem. IIE Transactions 1987;19:67-72.

[11] Dixon PS, Silver EA. A heuristic solution procedure for the multi item single level limited capacity lot sizing problem. Journal of Operations Management 1981;2:23-39.

[12] Sarin SC, Chen CS. The machine loading and tool allocation problem in a flexible manufacturing system. International Journal of Production Research 1987;25:1081-94.

[13] Tang CS, Denardo EV. Models arising from a flexible manufacturing machine, Part I: minimization of the number of tool switches. Operations Research 1988;36:767-77.

[14] Tetzlaff UAW. A queueing network model for flexible manufacturing systems with tool management. IIE Transactions 1996;28:309-17.

[15] Avci S, Akturk MS. Tool magazine arrangement and operations sequencing on CNC machines. Computers \& Operations Research 1996;23:1069-81.

[16] Wysk RA, Davis RP, Tanchoco RMA. Machining parameter optimization with lot size considerations. AIIE Transactions 1980;12:59-63.

[17] Koulamas CP. Optimal lot sizing and machining economics. Journal of Operational Research Society 1990;41:943-52.

[18] Koulamas CP. Simultaneous determination of the cutting speed and lot size values in machining systems. European Journal of Operational Research 1995;84:356-70.

[19] Akturk MS, Avci S. Tool allocation and machining conditions optimization for CNC machines. European Journal of Operational Research 1996;94:335-48.

M. Selim Akturk is an Assistant Professor of Industrial Engineering at Bilkent University, Turkey. He holds a Ph.D. in Industrial Engineering from Lehigh University, USA, and B.S.I.E. and M.S.I.E. from Middle East Technical University, Turkey. His current research interests include hierarchical planning of large scale systems, production scheduling and advanced manufacturing technologies. His papers have been published in Computers \& Operations Research, Computers in Industry, European Journal of Operational Research, Journal of Intelligent Manufacturing, IIE Transactions, International Journal of Advanced Manufacturing Technology, International Journal of Computer Integrated Manufacturing, International Journal of Production Research, and Production Planning \& Control.

Siraceddin Onen is a technical team leader at Razorfish Inc., Cambridge, MA. He received B.S.C.S. and B.S.I.E. degrees from Bosphorus University, Turkey and an M.S.I.E. from Bilkent University. His research interests include optimization theory, object oriented design and analysis, object oriented programming languages and management information systems. 